\title{
AdBlue Quality Control using Impedance Spectroscopy
}

\author{
Ahmed Fendri ${ }^{1}$, Paul Büschel ${ }^{1}$, Amine Abelkafi ${ }^{1}$, Olfa kanoun ${ }^{1}$, Andreas Buchholz ${ }^{2}$ \\ ${ }^{1}$ Chair of measurement and sensor Technology, Technische Universität Chemnitz, Germany \\ Ahmed.fendri@s2013.tu-chemnitz.de \\ ${ }^{2}$ Seuffer GmbH \& Co.KG
}

\begin{abstract}
Europe Union has established certain rules in order to reduce the contribution of vehicle engines to the Nitrogen Oxide $\left(\mathrm{NO}_{\mathrm{x}}\right)$ in air. SCR (Selective Catalytic Reduction) is one of the methods adopted by the vehicle manufacturers to meet the Europe union rules. It reduces $\mathrm{NO}_{x}$ emissions using AdBlue in the exhaust flow. AdBlue is an aqueous solution made with $32.5 \%$ pure urea and $67.5 \%$ de-ionized water. In order to be sure that systems are not compromised, it is essential to control the AdBlue quality in the tank. This work demonstrates that it's possible to determine the quality of AdBlue depending on the Urea. The results show the influence of urea concentration on the electrochemical behavior of the measured solution.
\end{abstract}

Key words: Nitogen Oxide, Selective Catalytic Reduction, Adblue, Impedance Spectroscopy

\section{Introduction}

Scientific researches demonstrate that $\mathrm{NO}_{x}$ emissions contribute to the formation of fine particles and ozone smog that cost billions annually from illnesses, deaths and environmental problems [1, 2]. The selective catalytic reduction (SCR technology) is one of the most used techniques for meeting the emission standards; it consists on converting the nitrogen oxides into innocuous nitrogen and water in the presence of a reducing agent: AdBlue. The quality of adblue should be controlled against the adulteration (use of AdBlue from a non-conforming source) and dilution of Urea with other substances. Detection of AdBlue quality is crucial for scientific research as it's an aqueous solution scalable for adulteration especially with water. Many works were done in order to detect the quality of AdBlue which are based on the ultrasonic, acoustic resonance, optical refractometry and near Infrared technologies [57]. Other works are focusing on the determination of urea concentration in an aqueous solution using the dielectric properties of the solution; it was shown that the relative dielectric permittivity increases if the urea concentration increases [8].

Electrochemical Impedance Spectroscopy (EIS) is a powerful technique which can be used to characterize liquids by measuring the impedance as a function of frequency. In our work, it was investigated whether the impedance spectroscopy is a suitable method for concentration monitoring.

\section{Experimental description}

For the first measurements we used the commercial Adblue (from a petrol station) which has a concentration $\mathrm{C}_{\text {AdBlue }}$ in the range of 31.8 to 33.2 wt- $\%$.

In these measurements the effects of variations of concentration were investigated. The impedance was recorded over a frequency range of $40 \mathrm{~Hz}$ to $110 \mathrm{MHz}$ using the Agilent 4294A Impedance analyzer. The measurements were made using a stainless steel cylindrical electrode made from X6CrNiTi18-10 alloy. The concentration is varied from 0 wt- $\%$ to $32.5 \mathrm{wt}-\%$. We supposed that the initial concentration is $32.5 \mathrm{wt} \%$ and we prepared the other concentration by dilution with de-ionized water.

\section{Results and discussion}

The measurements results show the effect of concentration on the impedance spectra. The charge transfer resistance decreases when the concentration increases, instead the Cell capacitance increase when the concentration increases. The capacitance of the electrode depends on the electrode geometry and the electrolyte in between the electrode surfaces. 


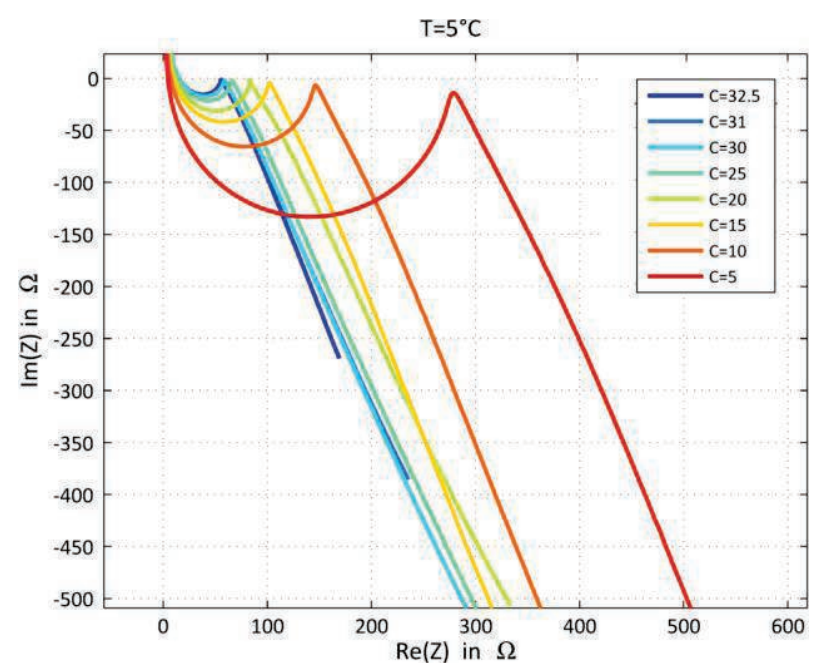

Fig.1 Nyquist plots for different concentration at $\mathrm{T}=5^{\circ} \mathrm{C}$

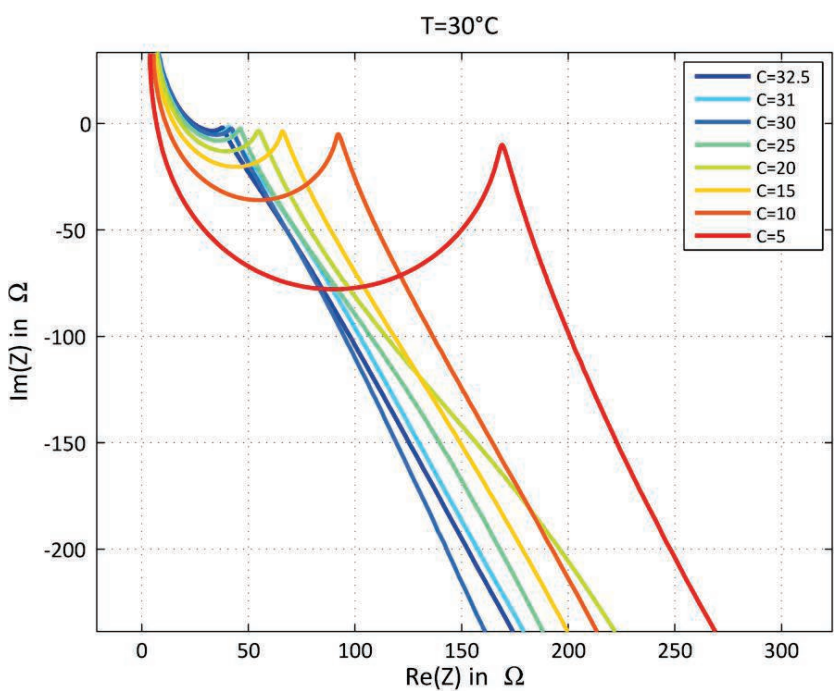

Fig.2 Nyquist plots for different concentration at

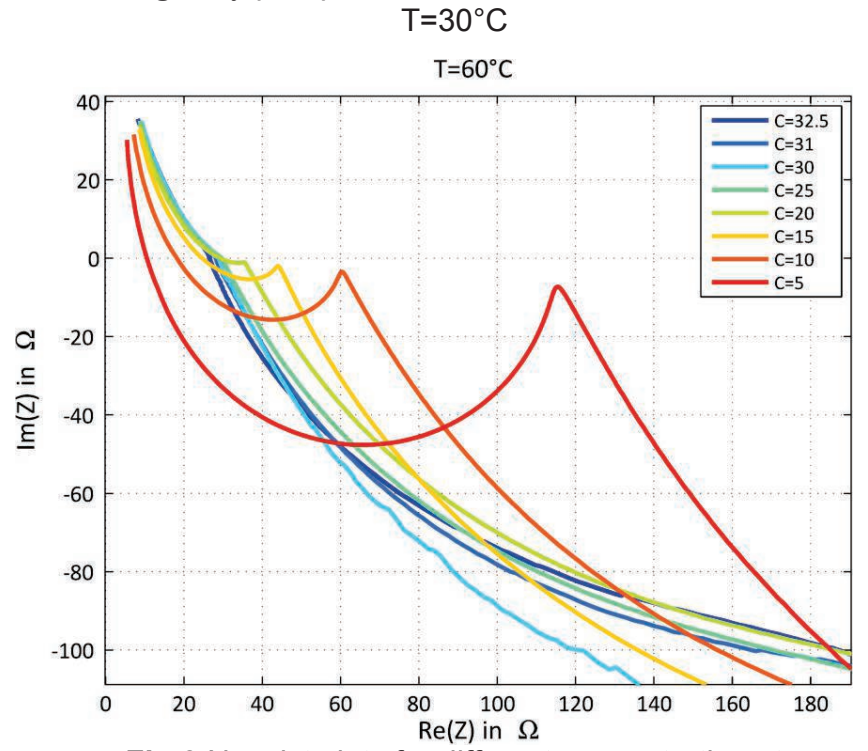

Fig.3 Nyquist plots for different concentration at $\mathrm{T}=60^{\circ} \mathrm{C}$
The impedance data were fitted using the equivalent circuit presented in Fig.4, It shows the principal phenomena involved in the electrochemical system and it consists of:

- $L_{\text {para: }}$ Inductance caused by electrode geometry and measurement setup

- $\mathrm{R}_{\mathrm{ohm}}$ : ohmic resistance of the cables

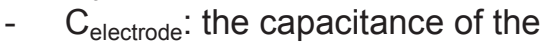
electrode

- Relectrolyte: The resistance of an ionic solution

- $\quad \mathrm{C}_{\mathrm{dl}}$ : double layer capacitance

- $\mathrm{R}_{\mathrm{ct}}$ : charge transfer resistance

- Warburg diffusion

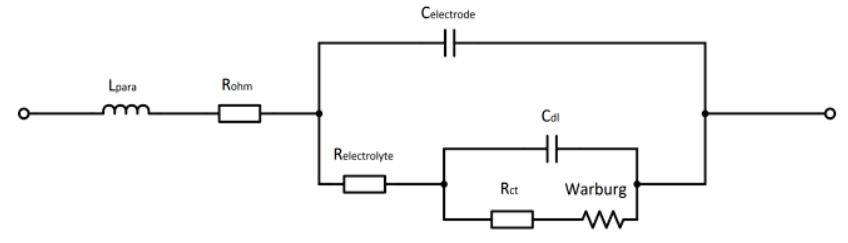

Fig.4 equivalent circuit

Fig.5 shows the variation of the cell capacitance $\left(\mathrm{C}_{\text {electrode }}\right)$ depending on the Adblue concentration and the temperature, $\mathrm{C}_{\text {electrode }}$ increases linearly when the concentration increases and it decreases when the temperature increases. We can use this relation to determine the adblue concentration in a solution at a constant temperature.

The concentration effect can be explained by the higher dielectric constant of some ionic electrolytes compared to pure water (the dielectric constant increases when the concentration increases). Also the temperature characteristic can be explained by the reduction of the dielectric constant of ionic solutions, due to decreased polarization by thermal influence.

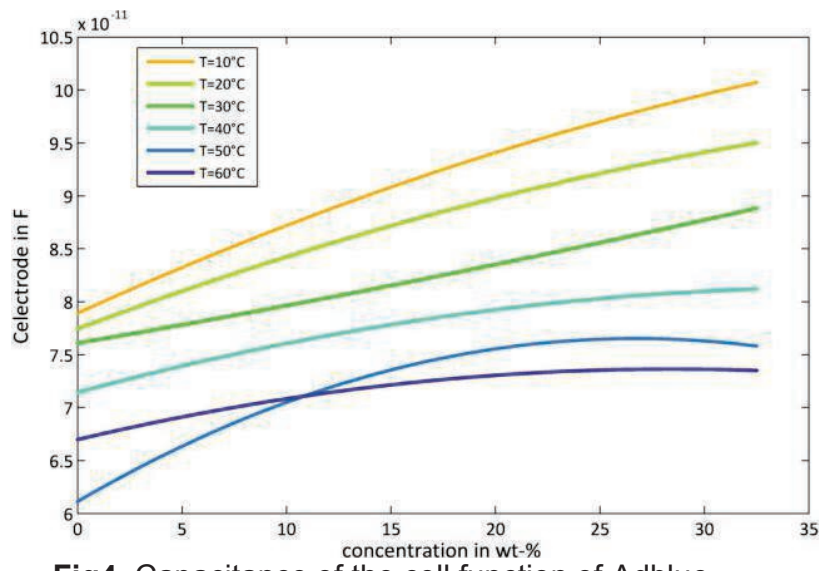

Fig4. Capacitance of the cell function of Adblue concentration at different temperature

The Kohlrausch's law shows that the equivalent conductivity of an electrolyte is equal to the sum of the conductances of charge carriers (anions and cations). The number of charge in an electrolyte represents the concentration of ions 
in the solution. Thus the conductivity is directly proportional to concentration. An increase in the concentration leads to increase in the conductivity [9].

An increase in a solution's temperature of electrolytic solutions (or liquids) will cause a decrease in its viscosity and an increase in the mobility of the ions in solution. An increase in temperature may also cause an increase in the number of ions in solution due to dissociation of molecules. As the conductivity of a solution is dependent on these factors then an increase in the solution's temperature will lead to an increase in its conductivity. [9]

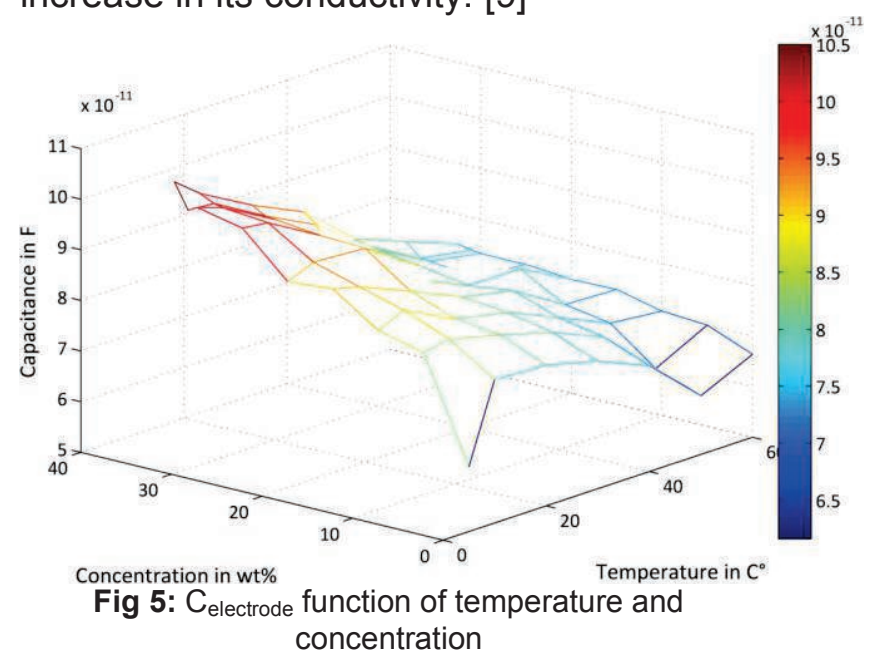

In this investigation the electrode geometry is constant but the electrolyte concentration and temperature were changed. As shown in figure 6 the value of the capacitance obtained from the fitting of the model for low concentrations ( $5 \mathrm{wt}-\%$ ) matches the estimated value of $73 \mathrm{pF}$ for pure water at $20{ }^{\circ} \mathrm{C}$. The influence of an increasing temperature is a decrease of capacitance. This temperature characteristic is caused by the reduction of the dielectric constant of water [8] and ionic solutions, due to decreased polarization by thermal influence.

\section{References}

[1] Huss group, Nitrogen Oxides $\left(\mathrm{NO}_{\mathrm{x}}\right)$

[2] Environmental Protection Agency,NC 27711, how nitrogen oxides affect the way we live and breathe

[3] DieselNet, Regulatory Framework

[4] Commission regulation (EU) No 459/2012, Official Journal of the European Union

[5] Accostic resonance based urea quality sensor, patentbuddy No 2008/0208,371
[6] Low cost optical refractometers for hobbyists and small to medium sized businesses, Global Water [7] G.W. small, Determination of Urea in aqueous samples by near Infrared spectroscopy

[8] A. satz, W. Granig, D. Tumpold and F.Reininger, sensors \& Transducers 120, Issue 9, Sept 2010, pp. 27-37

[9] http://fr.wikipedia.org/wiki/AdBlue 